\title{
INTENSITIES AND SPECTRA OF SEVERAL GALACTIC X-RAY SOURCES
}

R. BATSTONE, B.COOKE, R. GOTT, and E.STEWARDSON University of Leicester, Leicester, England

No text nor Summary was communicated by the Authors. 